\title{
Hétérozygotie des isolats de Phytophthora infestans; conséquences dans l'apparition des races physiologiques
}

\author{
Françoise LE GRAND-PERNOT \\ avec la collaboration technique de Fernande DutFor \\ Université de Paris-Sud, Laboratoire de Cryptogamie, associé au C.N.R.S.-U.A. 86, Bâtiment 402, F 91405 \\ Orsay
}

\begin{abstract}
Aucun des $\mathbf{4}$ isolats originels de Phytophthora infestans (Mont.) de Bary étudiés, n'a exprimé spontanément de nouvelles virulences, hormis dans un cas la virulence 4 . En revanche, celles-ci peuvent se manifester d'emblée chez des individus issus de la reproduction sexuelle, ou obtenus à la suite de l'action de la parafluorophénylalanine. Ces faits semblent exclure l'intervention de mutations. En conclusion, le démasquage de virulences nouvelles, chez les lignées avirulentes, résulte du remaniement, ou de l'isolement, d'allèles de virulence récessifs, déjà présents à l'état hétérozygote dans ces lignées.
\end{abstract}

Mots clés additionnels : Reproduction sexuelle, virulence récessive, parafluorophénylalanine.

Natural heterozygosity of Phytophthora infestans (Mont.) de Bary and its consequences for the appearance of physiologic races.

\begin{abstract}
Four original isolates of Phytophthora infestans studied expressed no new spontaneous virulence, except virulence 4 for one strain. However new virulences appeared immediately after sexual reproduction or after parafluorophenylalanine treatment of the original strains. From these observations we exclude spontaneous mutation as a mechanism generating new virulences, and conclude that these new virulences arising from avirulent strains must come from rearrangement or isolation of recessive virulence alleles, previously present within a heterozygous genome.
\end{abstract}

Additional key words : Sexual reproduction, recessive virulence, parafluoropheylalanine.

\section{INTRODUCTION}

Par hybridation interspécifique entre Solanum tuberosum Bauhin et Solanum demissum Lindl., la pomme de terre a été dotée d'une résistance à caractère monogénique qui renforçait les mécanismes de défense de la plante et qui lui donnait une protection totale vis-à-vis de certaines races de Phytophthora infestans. En introduisant successivement plusieurs gènes $\mathrm{R}$ dans le génome $S$. tuberosum, les sélectionneurs espéraient enrayer la maladie. Mais les populations de $P$. infestans ont toujours répondu à la pression exercée sur elles par les gènes $R$, en modifiant leur composition. Les races virulentes apparaissent plus ou moins tôt prédominantes, ou même éliminent totalement les autres éléments de la population (CHEVAUGEON, 1979).

La nature diploïde du cycle végétatif est maintenant bien établie (SHAw, 1983), il nous a donc semblé inté- ressant d'examiner les structures génétiques possibles des isolats naturels pour un certain nombre de caractères (croissance et sporulation à $16{ }^{\circ} \mathrm{C}$, développement et morphotype à $10^{\circ} \mathrm{C}$ ). Dans tous les cas nous avons démontré une hétérozygotie partielle (LE GRAND-PERNOT, 1986b). De même, TOOLEY \& FRY (1983) ont montré que la plupart des isolats testés de $P$. infestans étaient hétérozygotes pour de nombreux gènes. Peut-il en être ainsi pour les gènes qui contrôlent les caractères de virulence ? Parvenir à une telle réponse permettrait aussi d'attribuer aux allèles de virulence un caractère dominant ou récessif.

Nous avons donc étudié le comportement de descendants de première génération, issus de croisements entre lignées virulentes et avirulentes, ou entre lignées avirulentes, sur une gamme d'hôtes différentielle mettant en jeu les gènes majeurs de résistance $R 1, R 2, R 3$ et R4. Nous avons cherché dans un deuxième temps à haploïdiser les lignées parentales par l'action de la 
parafluorophénylalanine (pfa). Les ségrégants haploïdes sont ensuite testés sur la même gamme d'hôtes.

\section{MATÉRIEL ET MÉTHODES}

\section{A. Les souches}

Les souches parentales (A, D, St et Cv) et certains de leurs descendants issus directement des différentes combinaisons de croisements, décrits et étudiés précédemment ont été utilisés (LE GRAND-PERNOT, 1986b). Ils sont entretenus sur milieu $C$ gélosé (LE GRANDPERNOT, 1981). Le maintien d'une bonne activité parasitaire générale est assuré par un passage périodique sur tranches de tubercules de variétés réceptives à toutes les races de $P$. infestans, quelle que soit leur virulence. Ces variétés sont Bintje ou BF15.

\section{B. Identification de quelques caractères de virulence}

La constitution d'une gamme d'hôtes différentielle comprenant des variétés porteuses ou non d'un gène de résistance spécifique différent, permet de déterminer le pouvoir pathogène des souches étudiées (parentales et mono-oospores de $1^{\text {re }}$ génération). Nous présentons dans le tableau 1 les types d'interactions races/variétés que nous avons observées au cours des inoculations avec les souches parentales. Les 5 essais

\section{TABLEAU 1}

Exemple d'interactions entre les souches parentales de races variées et les différentes variétés de pomme de terre testées. Example of interactions between parental strains of different races and several varieties of tested potatoes.

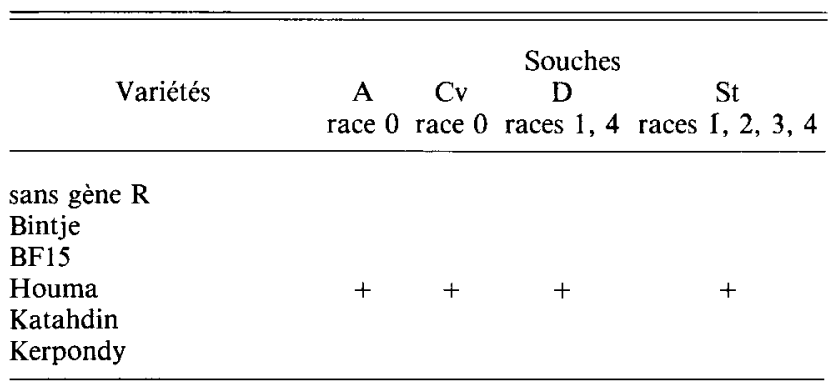

avec gène $\mathrm{R} 1$

Black 1

Claudia

Kennebec

Maritta

\begin{tabular}{|c|c|c|c|c|}
\hline $\begin{array}{l}\text { avec gène R2 } \\
\text { Black } 2\end{array}$ & - & - & - & + \\
\hline $\begin{array}{l}\text { avec gène R3 } \\
\text { Arka }\end{array}$ & & & & \\
\hline Black 3 & - & - & - & + \\
\hline
\end{tabular}

avec gène $\mathrm{R} 4$

Black 4

\section{$+$}

$+$

- interaction incompatible, le champignon ne se développe pas.

+ interaction compatible, le champignon envahit le tubercule.

- incompatible interaction, no mycelial growth.

+ compatible interaction, tuber invasion. indépendants d'inoculations sur folioles ont conduit généralement à des échecs renouvelés et nous ont empêché de caractériser la plupart des souches. En revanche les 6 expériences effectuées sur tranches de tubercules permettent de qualifier ou de préciser un ou plusieurs caractères de virulence. Les inoculations ont été réalisées $2,5,14,16,19$ et 25 mois après l'isolement des descendants selon la méthode suivante : des tubercules épluchés sont désinfectés superficiellement dans l'alcool éthylique absolu, puis flambés et enfin découpés en tranches d'environ $8 \mathrm{~mm}$ d'épaisseur. Deux tranches de tubercule sont inoculées avec une suspension de sporocystes ou une bouture mycélienne, puis rassemblées, face infectée contre face infectée, et déposées en boîte de Petri dans laquelle on a eu soin de mettre un coton imbibé d'eau. Par souche et par variété on réalise de 3 à 6 inoculations. L'ensemble est porté en chambre de culture réglée à $16{ }^{\circ} \mathrm{C}$. La lecture du test a lieu de 4 à 7 jours plus tard. Lorsqu'il existe une interaction compatible, c'est-à-dire que la souche est virulente, le mycélium réapparaît en surface après avoir traversé la tranche supérieure du tubercule; lorsque l'interaction est incompatible, c'est-à-dire que l'infection a échoué, les tranches de tubercule ont une apparence parfaitement saine et sont identiques à celles servant de témoin.

\section{Action de la DL parafluorophenylalanine (pfa)}

Trois des 4 souches parentales (A, D et $\mathrm{Cv}$ ) sont repiquées sur milieu $\mathrm{C}$ gélosé additionné de DL parafluorophénylalanine $(\mathrm{pfa})$ à des concentrations finales de 0,$4 ; 1 ; 10$ et $100 \mathrm{mg} / \mathrm{l}$. Vingt huit boîtes de Petri sont réalisées par souche et par dose. Des filaments prélevés dans les secteurs et plages formés sur quelques-unes des boîtes contenant de la pfa, et des boutures prises dans des thalles obtenus en l'absence de pfa sont repiqués sur milieu $\mathrm{C}$ additionné d'une solution d'antibiotiques (100 p.p.m. de Pimafucine, 50 p.p.m. de Polymixine et 50 p.p.m. de Penicilline) puis testés entre 2 tranches de tubercule de variétés porteuses ou non des gènes R1 ou R2 ou R3 ou R4.

\section{RÉSULTATS}

$P$. infestans est un champignon hétérothallique, mais la présence simultanée des 2 souches complémentaires induit réciproquement chez chacun des partenaires la différenciation des gamètes mâles et femelles. Leur confrontation aboutit à la formation de produits d'autofécondation des 2 sortes et d'hybrides mélangés et de morphologie identique.

L'étude d'un certain nombre de caractères comme la croissance et la sporulation à $16^{\circ} \mathrm{C}$, le développement et le comportement à $10^{\circ} \mathrm{C}$ (LE GRANDPERNOT, 1986b), le signe de compatibilité sexuelle (LE GRAND-PERNOT, 1983), nous a permis d'envisager dans la plupart des cas l'origine des descendants (LE GRAND-PERNOT, 1983), dont nous tenons compte pour présenter les résultats qui suivent concernant l'identification de quelques caractères de virulence. 
Par des inoculations répétées et réparties sur 25 mois, nous avons ainsi pu déterminer et contrôler le pouvoir pathogène des lignées parentales et de leur descendance autofécondée ou hybride.

\section{A. Comportement des parents}

Toutes les souches parentales ont gardé leur propriété originelle au cours de 25 mois de tests du pouvoir pathogène. Ainsi les souches parentales avirulentes $\mathrm{A}$ et $\mathrm{Cv}$ n'ont jamais révélé de caractère de virulence au cours des 2 années de tests. La souche St a conservé depuis l'origine les virulences $1,2,3$ et 4 . La souche $\mathrm{D}$, qui à sa réception ne possédait que la virulence 1, a manifesté quelques mois après sa mise en culture, et bien avant l'obtention des descendants, la virulence 4 . Ceci est à mettre en parallèle avec l'apparition très fréquente de la virulence 4 dans les populations naturelles (TOXOPEUS, 1959).

\section{B. Analyse des différentes F1}

\section{Descendance autofécondée (tabl. 2a)}

Les 4 descendants issus de l'autofécondation de la souche $\mathrm{St}$, de virulences $1,2,3$ et 4 ont exprimé, comme St, toutes ces virulences en même temps. L'unique descendant originaire de A est avirulent comme son parent. Le descendant obtenu à partir de la souche D seule, a un spectre d'hôtes plus élargi que celui de son parent, puisqu'il est capable d'attaquer aussi les variétés porteuses de gènes de résistance 2 et 3.

\section{Descendance hybride (tabl. 2b)}

Les hybrides formés à la suite du croisement entre les souches $\mathrm{A}$ et $\mathrm{St}$ ne se comportent pas tous de la même façon sur la gamme d'hôtes différentielle : certains sont avirulents, d'autres possèdent une ou plusieurs virulences du parent $\mathrm{St}$, mais à aucun moment la virulence 1 , présente chez ce même parent, ne s'est manifestée d'emblée chez les descendants hybrides; en revanche, elle apparaît toujours après autofécondation de la souche St.

De même, les 2 hybrides originaires des souches A et $\mathrm{D}$ ne présentent pas non plus la virulence 1 . En revanche, ce croisement peut révéler l'expression de nouvelles virulences; les virulences 2 et 3 déjà mises en évidence lors de l'autofécondation de la souche D.

\section{Descendance dont l'origine est mal définie (tabl. 2c)}

Qu'il y ait eu autofécondation ou hybridation lors de la confrontation des souches $\mathrm{A}$ et $\mathrm{CV}$ il importe de constater que des souches avirulentes peuvent engendrer une descendance dotée d'une ou plusieurs virulences, que le ou les parents n'ont jamais exprimées.

Enfin, dans un cas nous avons pu déceler une souche hétérocaryotique qui présentait des noyaux d'origine $A$ et St (LE GRAND-PERNOT, 1983). Cet hétérocaryon testé sur 14 mois n'a jamais attaqué les variétés porteuses de gènes $R$.

\section{TABLEAU 2}

Identification des caractères de virulence chez les descendants autofécondés ou hybrides.

Identification of virulence characters of self-fertilized or hybrid progenies.

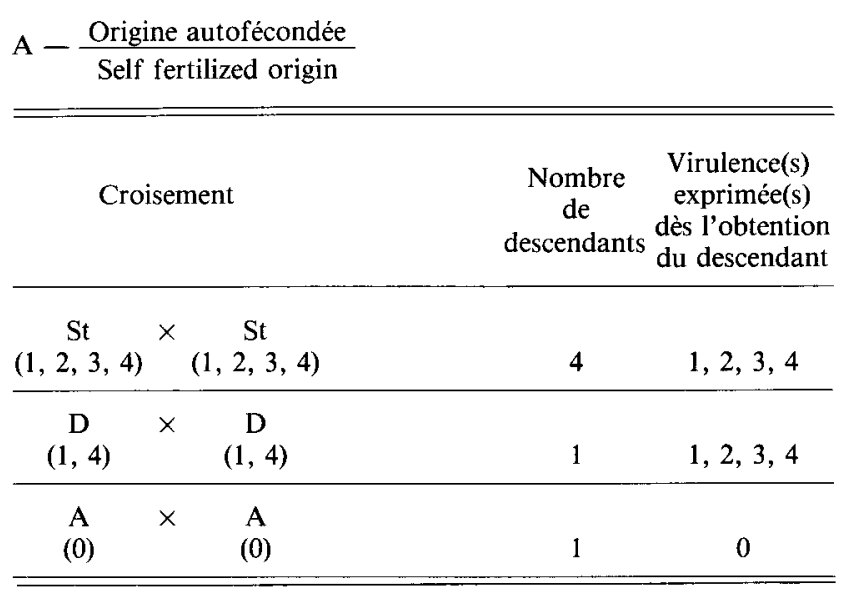

B - Origine hybride

hybrided origin

\begin{tabular}{ccccc}
\hline & & & & \\
A & $\times$ & St & 4 & 0 \\
$(0)$ & & $(1,2,3,4)$ & 3 & 2 \\
& & 2 & 3 \\
& & 1 & 2,3 \\
& & 1 & 2,4 \\
& & 1 & 3,4 \\
& & 3 & $2,3,4$ \\
\hline A & $\times$ & D & 1 & 3,4 \\
$(0)$ & & $(1,4)$ & 1 & $2,3,4$ \\
\hline
\end{tabular}

C - Origine non définie et autre situation

non definited origin and other situation

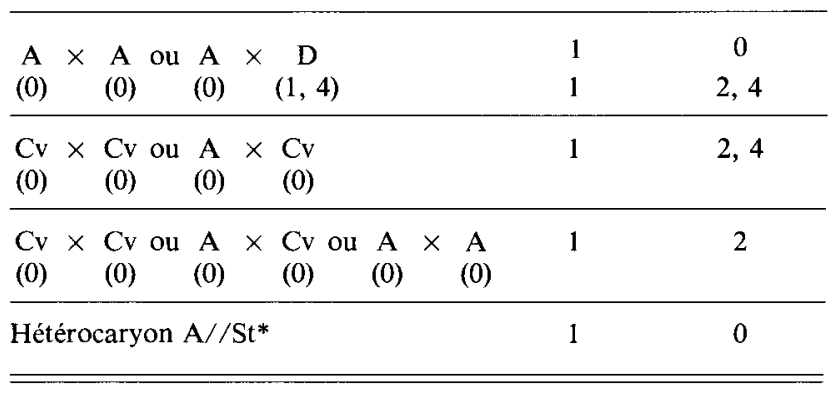

(*) L'hétérocaryon A//St est testé sur 14 mois.

\section{Evolution des souches monospores dans le temps}

Si la majorité des souches sont restées stables au cours des inoculations répétées quelques-unes ont manifesté, à des moments variés et après plusieurs repiquages en boîte de Petri, une ou plusieurs virulences (tabl. 3).

Ainsi 2 hybrides du croisement A par St qui présentent des différences au cours du $1^{\text {er }}$ test d'inoculation finissent après 16 mois par acquérir les mêmes virulences que celles de leur parent St. La souche hétérocaryotique avirulente, au moins les 14 premiers mois, mettra près d'un an avant de se comporter comme la souche St. Si la virulence 1 n'est jamais apparue chez 
TABLEAU 3

Apparition précoce ou tardive de certains caractères de virulence chez des descendants originaires de confrontations différentes. Early and late appearance of some virulence characters with progenies from different crosses.

\begin{tabular}{|c|c|c|c|c|c|c|}
\hline \multirow{2}{*}{$\begin{array}{c}\text { Origine } \\
\text { du descendant }\end{array}$} & & \multicolumn{5}{|c|}{ Virulences exprimées } \\
\hline & $\begin{array}{l}\text { dès l'isolement } \\
\text { de la souche } \\
\text { mono-oospore }\end{array}$ & \multicolumn{5}{|c|}{ après un délai (en mois) de } \\
\hline hybride $(\mathrm{A} \times \mathrm{St})$ & $2,3,4$ & & $1(2,3,4)$ & 1234 & 1234 & 1234 \\
\hline hybride $(\mathrm{A} \times \mathrm{St})$ & 3 & $1(3)$ & $4(1,3)$ & $2(134)$ & 1234 & 1234 \\
\hline hétérocaryon $(\mathrm{A} / / \mathrm{St})$ & 0 & - & - & 2,3 & $1(2,3)$ & $4(1,2,3)$ \\
\hline autofécondée $(A)$ ou hybride $(A \times D)$ & 0 & - & - & - & - & 2,4 \\
\hline
\end{tabular}

les hybrides dès leur obtention, elle peut toutefois apparaître tardivement chez certains d'entre eux. Bien que l'origine soit mal définie par le dernier représentant de ce tableau il semble que l'apparition tardive de certaines virulences ne soit pas le privilège d'une association particulière.

\section{Action de la DL parafluorophénylalanine (pfa)}

L'incorporation de la pfa dans le milieu de culture perturbe le développement des souches $\mathrm{A}, \mathrm{D}$ et $\mathrm{Cv}$, et favorise dans de nombreux cas la prolifération bactérienne, ce qui réduit au quart $(7 / 28)$ le nombre de bô̂tes d'expérimentation exploitables. Les doses de $0,4 \mathrm{mg} / \mathrm{l}$ et $\mathrm{de} 1 \mathrm{mg} / \mathrm{l}$ altèrent peu leur élongation radiale, en revanche elles réduisent la quantité de mycélium aérien et de sporocystes. A $10 \mathrm{mg} / \mathrm{l}$ la croissance et la sporulation sont très fortement ralenties et tendent à s'arrêter après 8 jours d'expérience. Enfin, aucun thalle ne se forme à partir de boutures déposées sur un milieu contenant $100 \mathrm{mg} / \mathrm{l}$. Après 18 jours de culture : dans 3 cas sur 7 , la souche $A$ en présence de $0,4 \mathrm{mg} / \mathrm{l} \mathrm{de}$ pfa montre une plage où le mycélium est plus abondant ; les souches $\mathrm{D}$ et $\mathrm{Cv}$ réagissent à la présence de $10 \mathrm{mg} / \mathrm{l}$ de pfa en formant, au-delà de la zone d'arrêt de croissance, 1 ou 2 secteurs irréguliers sur 2 ou 3 des 7 boîtes analysables.

Plages et secteurs sont testés sur la même gamme d'hôte différentielle (tabl. 4). Les thalles sont capables d'exprimer de nouvelles virulences. Ainsi, 1 des 3 secteurs de la souche $\mathrm{Cv}$ (s3) manifeste les virulences $1,2,3$ et 4 ; de même 1 des 3 secteurs de la souche D $\left(s^{\prime} 2\right)$ est devenu compatible avec toutes les variétés testées. Enfin, 2 plages (p11, p13) révélées indépendamment sur 2 thalles de la souche A ont exprimé respectivement les virulences 3,4 et $2,3,4$.

\section{DISCUSSION}

L'apparition de nouvelles races chez $P$. infestans a surtout été étudiée au cours du cycle végétatif. Les observations in vitro et en champ de ce phénomène ont conduit à des interprétations variées.

Ainsi pour GALLEGLY \& EICHENMULLER (1959) des mutations spontanées seraient à l'origine de l'expres-

\section{TABLEAU 4}

Identification du pouvoir pathogène des plages et secteurs apparus chez les souches $A, C v$ et $D$ cultivées en présence de parafluorophénylalanine $(p f a)$.

Pathogenicity identification of dense mycelial area and sectors appeared with strains $A, C v$ and $D$, grown with parafluorophenylalanine.

\begin{tabular}{|c|c|c|c|c|c|c|}
\hline & \multirow{2}{*}{ Souches } & \multicolumn{5}{|c|}{$\begin{array}{l}\text { Virulences exprimées } \\
\text { sur les génotypes des plantes }\end{array}$} \\
\hline & & I & R1 & $\mathrm{R} 2$ & R3 & R4 \\
\hline \multirow{4}{*}{$\mathrm{CV}$} & $\begin{array}{l}\text { sans pfa } \\
\text { avec } 10 \mathrm{mg} / 1 \mathrm{pfa}:\end{array}$ & + & - & - & - & - \\
\hline & secteur S1 & + & - & - & - & - \\
\hline & secteur S2 & + & - & - & - & - \\
\hline & secteur S3 & + & + & + & + & + \\
\hline \multirow{4}{*}{$\mathrm{D}$} & $\begin{array}{l}\text { sans } \mathrm{pfa} \\
\text { avec } 10 \mathrm{mg} / \mathrm{pfa} \text { : }\end{array}$ & + & + & - & - & + \\
\hline & secteur $S^{\prime} 1$ & + & + & - & - & + \\
\hline & secteur $S^{\prime} 2$ & + & + & + & + & + \\
\hline & secteur $S^{\prime} 3$ & + & + & - & - & + \\
\hline \multirow{4}{*}{ A } & $\begin{array}{l}\text { sans pfa } \\
\text { avec } 4 \mathrm{mg} / \mathrm{l} \mathrm{pfa}\end{array}$ & + & - & - & - & - \\
\hline & plage p11 & + & - & - & + & + \\
\hline & plage p12 & + & - & - & - & - \\
\hline & plage p13 & + & - & + & + & + \\
\hline
\end{tabular}

+ virulent ; - avirulent.

sion de nouvelles virulences, hypothèse que WILDE (1961) a pu confirmer en soumettant des souches à l'effet mutagène des radiations ultra-violettes.

L'hétérocaryose, que WILDE (1961), LEACH \& RICH (1969), Denward (1970), Malcolmson (1970) ont mis en évidence après avoir mélangé des souches présentant des virulences différentes expliquerait aussi l'obtention de races nouvelles. Mais les caractères de virulence, comme nos expériences le laissent entendre et comme d'autres auteurs l'ont montré in vitro et en champ (TOXOPEUS, 1959), sont sujets à des variations spontanées, ce qui a conduit à douter du rôle réel de l'hétérocaryose dans ces phénomènes, même si son intervention est mieux démontrée par D'YAKOV \& KUZOVNIKOVA (1974) qui ont fait appel à des marqueurs plus stables, en particulier des résistances à des antibiotiques. 
DENWARD (1970) expliquerait l'apparition de nouvelles virulences par l'existence d'une structure extranucléaire, autorépliquante, temporaire à large diffusion - du type plasmide (BRODA, 1979) - qui pourrait être incorporée, sous certaines conditions, dans le génome des souches.

LEACH \& RICH (1969) supposent un changement d'activité du génome, en réponse à une modification du milieu interne : des gènes présents dans certaines souches, mais non actifs, seraient déréprimés à la faveur du mélange de 2 protoplasmes (anastomoses).

Mais toutes ces hypothèses ont été émises d'une part à propos du cycle végétatif et d'autre part le plus souvent dans le contexte d'un organisme haplö̈de. Il est certain que la démonstration de la diploïdie des représentants du genre Phytophthora et l'obtention de nouvelles virulences par l'intermédiaire de la reproduction sexuelle contribuent à formuler ou préciser certaines hypothèses.

Si les gènes de virulence nucléaires (NIEDERHAUSER, 1961 ; ROMERO \& ERWIN, 1969) et indépendants (ROMERO \& ERWIN, 1969) sont récessifs et que les isolats sont hétérozygotes à ces loci, on doit observer une descendance hétérogène. Par le jeu de ces recombinaisons méiotiques entre noyaux $\mathrm{AV} / /$ av puis l'association au hasard des gamètes $\mathrm{AV}$ et av formés, il sera possible d'obtenir des descendants homozygotes récessifs av//av qui seront devenus virulents. Cette situation reste vraie que les gamètes aient même origine ou qu'ils soient d'origine différente, c'est-à-dire que la souche mono-oospore soit d'origine autofécondée ou hybride (tabl. 2a, b, c).

Dans le cadre de cette hypothèse nous pouvons ébaucher la structure génétique des souches parentales vis-à-vis de ces caractères.

Analysons dans un premier temps les résultats présentés dans le tableau $2(\mathrm{a}, \mathrm{b}, \mathrm{c})$. Lors du croisement de 2 souches avirulentes $\mathrm{A}$ et $\mathrm{Cv}$ (tableau 2c), l'expression des virulences 2 et 2,4 suppose qu'un partenaire, ou les deux possèdent une structure hétérozygote pour les caractères 2 et 4 . Les deux parents pourraient donc avoir les génotypes suivants :

$$
\text { A : } \frac{\text { AV2 }}{\text { av2 }} \frac{\text { AV4 }}{\text { av4 }} \quad \text { CV }: \frac{\text { AV2 }}{\text { av2 }} \frac{\text { AV4 }}{\text { av4 }}
$$

Si peu de descendants ont une parenté avec $\mathrm{Cv}$, limitant momentanément sa structure à ce stade, d'autres, en revanche, en ont avec A, parmi eux, les 2 hybrides formés à partir de $\mathrm{A}$ et $\mathrm{D}$ (tabl. 2b), qui ont manifesté 1 ou 2 virulences dont $A$ et $D$ sont dépourvus. Ainsi les caractères de virulence 2 et 3 se présenteraient sous la forme hétérozygote chez ces 2 souches. Cette structure semble être confirmée pour le parent $D$, puisque le descendant né de l'autofécondation de D (tabl. 2a) exprime aussi ces 2 caractères. Ainsi les génotypes de $\mathrm{A}$ et de $\mathrm{D}$ seraient à ce stade de réflexion :

$$
\text { A : } \frac{\text { AV2 }}{\overline{\text { av2 }}} \frac{\text { AV3 }}{\text { av3 }} \frac{\text { AV4 }}{\underline{\text { av4 }}} \quad \text { D : } \frac{\text { AV2 }}{\text { av2 }} \frac{\text { AV3 }}{\text { av3 }}
$$

Notons que la structure partielle de la souche $\mathrm{A}$, pré-établie pour les caractères 2 et 4 , est confirmée.

La virulence 4 apparaît d'emblée chez 4 des 5 descendants où la participation unique ou pour moitié de $\mathrm{D}$ est impliquée (tabl. $2 \mathrm{a}, \mathrm{b}, \mathrm{c}$ ). Son existence est justifiée par l'hybridation entre A (hétérozygote) et D (homozygote récessif pour ce caractère) (tabl. 2b), ou par l'autofécondation de $\mathrm{D}$ (homozygote récessif $\times$ homozygote récessif) (tabl. 2a) ou même par l'autofécondation de $\mathrm{A}$ (hétérozygote $\times$ hétérozygote) comme cela a pu être soupçonné (tabl. 2c).

En revanche, la virulence 1 présente aussi chez D et absente chez A est apparue, parmi ces mêmes descendants uniquement lors de l'autofécondation de la souche D (homozygote récessive pour ce caractère). L'avirulence observée dans les autres cas conduit à formuler 2 génotypes possibles en ce qui concerne la non expression du caractère 1 chez le parent $\mathrm{A}$ : homozygote dominant AV1//AV1 ou hétérozygote AV1//av1.

Ainsi 2 formules génotypiques sont possibles pour :

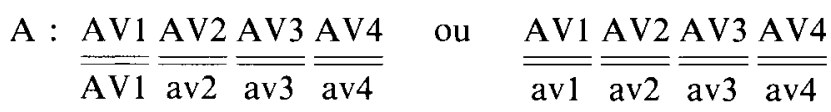

mais une seule caractérise le parent $D$, compatible avec les variétés $\mathrm{R} 1$ et $\mathrm{R} 4$

$$
\mathrm{D}: \frac{\mathrm{av1}}{\overline{\mathrm{av1}}} \frac{\mathrm{AV} 2}{\overline{\mathrm{av} 2}} \frac{\mathrm{AV} 3}{\overline{\mathrm{av3}}} \frac{\mathrm{av4}}{\overline{\mathrm{av4}}}
$$

L'ensemble des résultats obtenus lors de la confrontation de A par St s'accorde-t-il avec l'hypothèse d'allèles de virulence $1,2,3,4$ récessifs, et les 2 génotypes possibles de $\mathrm{A}$ ?

Dans la confrontation A par St, une majorité de descendants testés provient de l'hybridation entre les 2 lignées parentales (tabl. 2b) mais 5 d'entre eux (tabl. 2a) ont une origine autofécondée de la souche A (1 cas) ou de la souche St (4 cas). Or la Fl issue de l'autofécondation de $\mathrm{St}$ est homogène et exprime tous les caractères de virulence, ce qui renforce l'idée d'une structure homozygote pour ces caractères chez la souche St. L'unique descendant obtenu à partir du croisement A par A, et de phénotype avirulent, peut s'expliquer que $\mathrm{A}$ soit entièrement hétérozygote ou homozygote pour l'avirulence 1 et hétérozygote pour les virulences 2, 3 et 4. L'apparition chez les hybrides (tabl. 2b) des virulences 2, 3 et 4, seules ou associées, se conçoit aussi avec l'hétérozygotie du parent A pour ces 3 gènes, et l'homozygotie récessive correspondante pour le parent St. Mais, qu'aucun des 15 hybrides $(\mathrm{A} \times \mathrm{St})$ n'exprime d'emblée la virulence 1 tend à supposer que le parent $A$ possède plutôt, au locus du gène de virulence 1 , les 2 allèles d'avirulence correspondants. Ainsi l'hybridation entre un homozygote récessif av1//avl (St), et 1 homozygote dominant AV1//AV1 (A) conduit en $1^{\text {re }}$ génération à une descendance homogène hétérozygote $A V 1 / / a v 1$, exprimant le caractère dominant, à savoir l'incompatibilité avec les variétés porteuses du gène R1. Le génotype le plus probable de chacune de ces 2 souches serait donc :

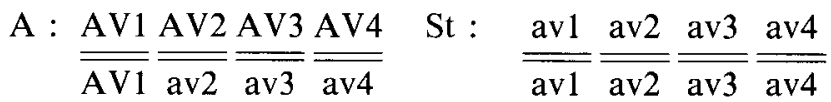

L'apparition d'une ou de plusieurs modifications tardives du pouvoir pathogène chez peu de descendants, 1 an et plus après leur obtention (tabl. 3), est constatée lorsque la participation des 2 génotypes parentaux est fortement soupçonnée et qu'une structure hétérozygote (ou hétérocaryotique dans 1 cas) s'est établie. Des évènements mutationnels ne peuvent 
être exclus, et l'expression de ces mutations récessives est facilitée par la structure hétérozygote du ou des gènes impliqués.

Nous n'avons pas été en mesure de déterminer le degré de ploïdie des noyaux contenus dans les plages et les secteurs obtenus en présence de pfa, mais les travaux de LHOAS (1961) sur Aspergillus niger Tregh, d'OlSON \& BORKHARDT (1978) sur Allomyces macrogynus (Emerson) Emerson et Wilson et surtout ceux de DAY \& JONES (1971) sur Ustilago violaces (Pers.) Fek, laissent penser que la pfa pourrait agir de la même façon sur $P$. infestans et provoquer la formation d'haploïdes par perte de chromosomes en une ou quelques étapes seulement.

S'il en est bien ainsi, certains ségrégants, haplö̈des de $P$. infestans possèderont alors à l'état unique 1 allèle de virulence donné ou l'allèle d'avirulence correspondant qui dans tous les cas pourra s'exprimer directement.

Si l'on considère l'ensemble des caractères de virulence on observe (tabl. 4) que tous les ségrégants d'une même origine ne sont pas identiques entre eux et à la souche qui leur a donné naissance. Les souches $\mathrm{A}$, D et $\mathrm{Cv}$ ne peuvent donc pas être homozygotes pour tous ces caractères, mais doivent posséder au moins pour un certain nombre d'entre eux une structure hétérozygote.

Ainsi les virulences 2,3 et 4 (tabl. 4) exprimées toutes ou partiellement ensemble par 2 ségrégants de la souche A suppose que cette dernière soit hétérozygote pour ces 3 caractères; et la non-expression de ces mêmes virulences par le parent A diplö̈de peut s'expliquer si l'on attribue aux allèles de virulences 2 , 3 et 4 une nature récessive. Le fait qu'aucune plage n'exprime la virulence 1 appuie de nouveau l'idée d'homozygotie pour ce caractère.

Le résultat obtenu pour 1 des 3 secteurs apparus chez la souche D traitée, corrobore la présence des allèles de virulence 2 et 3 à l'état hétérozygote chez l'isolat D témoin. De plus, le fait que les 3 secteurs aient conservé les caractéristiques de ce dernier vis-àvis des virulences 1 et 4 , attesterait de l'homozygotie de ces 2 caractères chez $D$ et renforcerait l'idée que ces virulences sont récessives.

Enfin la souche $\mathrm{Cv}$ avirulente, peut induire en présence de pfa, au moins 1 secteur capable d'exprimer de nouvelles potentialités, parmi lesquelles 2 , les virulences 2 et 4 , ont été déjà mises en évidence par croisement. Ainsi, l'hypothèse de la structure hétérozygote pour ces caractères est renforcée. Mieux cette nouvelle approche qui a permis en plus de détecter chez $\mathrm{Cv}$ les virulences 1 et 3 , apporte une précision sur la structure génétique possible de la souche, à savoir : l'hétérozygotie aussi pour les allèles de virulence 1 et 3 .

Les expériences permettant de confirmer nos résultats chez cette espèce ou d'autres espèces de ce genre sont peu nombreuses, seule MAIA (1986) a montré que le pouvoir pathogène de $P$. parasitica sur œillet était sous la dépendance de gènes récessifs.

La capacité de $P$. infestans à varier dans ses aptitudes parasitaires, constatée depuis l'intervention de l'homme pour endiguer la maladie, peut s'expliquer maintenant quel que soit le mode de variation adopté (LE GRAND-PERNOT, 1986a), par la présence, à l'état hétérozygote, de plusieurs allèles de virulence récessifs, dans le génome des isolats avirulents diploïdes.

Reçu le 9 décembre 1986. Accepté le 10 novembre 1987.

\section{RÉFÉRENCES BIBLIOGRAPHIQUES}

Broda P., 1979. Plasmids. Edited by N. H. Freeman and $\mathrm{C}^{\circ}$. Oxford and San Francisco, 197 p.

Chevaugeon J., 1979. Phytophthora infestans : un exemple d'intervention de l'homme sur la structure d'une espèce parasite. Bull. Soc. Fr., 126, Actual. Bot., 4, 21-44.

Day A. W., Jones J. K., 1971. P-fluorophenylalanine-induced mitotic haploidisation in Ustilago violacea. Genet. Res. Camb., 18, 299-309.

Denward T., 1970. Differentiation in Phytophthora infestans. II. Somatic recombination in vegetative mycelium. Hereditas, 66, 35-48.

Dyakov Y. T., Kuzovnikova T. A., 1974. Heterokaryosis in Phytophthora infestans (Mont.) de Bary. II. Genetic investigations. Mikol. Fitopatol. (en russe), 8, 81-89.

Gallegly M. E., Eichenmuller J. J., 1959. The spontaneous appearance of the potato race 4 character in cultures of Phytophthora infestans. Amer. Potato J., 36, 45-51.

Leach S. S., Rich A. E., 1969. The possible role of parasexuality and cytoplasmic variation in race differentiation in Phytophthora infestans. Phytopathology, 59, 1360-1365.

Le Grand-Pernot F., 1981. Facteurs influant sur le processus d'établissement de thalles monozoospores chez Phytophthora infestans. Can. J. Bot., 59, 836-841.

Le Grand-Pernot F., 1983. Tentative de domestication in vitro d'un champignon biotrophe Phytophthora infestans (Mont.) de Bary. Organisation du génome, ses conséquences. Thèse Doct. Etat, Orsay, France, $210 \mathrm{p}$.

Le Grand-Pernot F., 1986a. Quelques réflexions sur les sources de variations d'isolats Al de Phytophthora infestans (Mont.) de Bary. Agronomie, 6, 321-324.
Le Grand-Pernot F., 1986b. Les isolats de Phytophthora infestans (Mont.) de Bary sont-ils naturellement hétérozygotes ? Agronomie, $6(10), 877-883$.

Lhoas P., 1961. Mitotic haploidization by treatment of Aspergillus niger diploids with para-fluorophenylalanine. Nature, 190, 744.

Maia N., 1986. Contribution à l'étude de la transmission héréditaire de quelques caractères dans le genre Phytophthora. Thèse Doct. Etat, Orsay, France, 122 p.

Malcolmson J. F., 1970. Vegetative hybridity in Phytophthora infestans. Nature, 255, 971-972.

Niederhauser J. S., 1961. Genetic studies of Phytophthora infestans and Solanum species in relation to late blight resistance in the potato. Recent Advances in Botany, 491-497. Univ. Toronto Press. Torondo Canada.

Olson L. W., Borkhardt, 1978. Polyploidy and its control in Allomyces macrogynus. Trans. br. Mycol. Soc., 71, 65-76.

Romero S., Erwin D. C., 1969. Variation in pathogenicity of progeny from germinated oospores of Phytophthora infestans. Phytopathology, 59, 1310-1317.

Shaw D. S., 1983. The cytogenetics and genetics of Phytophthora. In Phytophthora its biology, taxonomy, ecology and pathology. Edited by D. C. Erwin, S. Bartnicki-Garcia and P. H. Tsao. Am. Phyto. Soc. St. Paul, 392 p.

Tooley P. W., Fry W. E., 1983. Genetic variation in Phytophthora infestans identified through isozyme analysis. Phytopathology, 73 (5), 827 .

Toxopeus H. J., 1959. Notes on the inheritance of field resistance of the foliage of Solanum tuberosum to Phytophthora infestans. Euphytica, 8, 117-124.

Wilde P., 1961. Ein Beitrag zur Kenntnis der Variabilität von Phytophthora infestans (Mont.) de Bary. Arch. Mikrobiol., 40, 163-165. 